\title{
A NEW IMAGING TECHNIQUE TO MONITOR DISEASE ACTIVITY IN SUSAC SYNDROME?
}

\author{
$\underline{\text { S. De Blauwe }}{ }^{1}$, A. De Vriese ${ }^{2}$, B. Lafaut ${ }^{3}$, J. Casselman4, G. Vanhooren¹.
}

${ }^{1} A Z$ Sint-Jan Brugge-Oostende AV, Neurology department, Brugge, Belgium.

${ }^{2} A Z$ Sint-Jan Brugge-Oostende AV, Internal Medicine department, Brugge, Belgium.

${ }^{3} \mathrm{AZ}$ Sint-Jan Brugge-Oostende AV, Ophtalmology department, Brugge, Belgium.

${ }^{4}$ AZ Sint-Jan Brugge-Oostende AV, Radiology department, Brugge, Belgium.

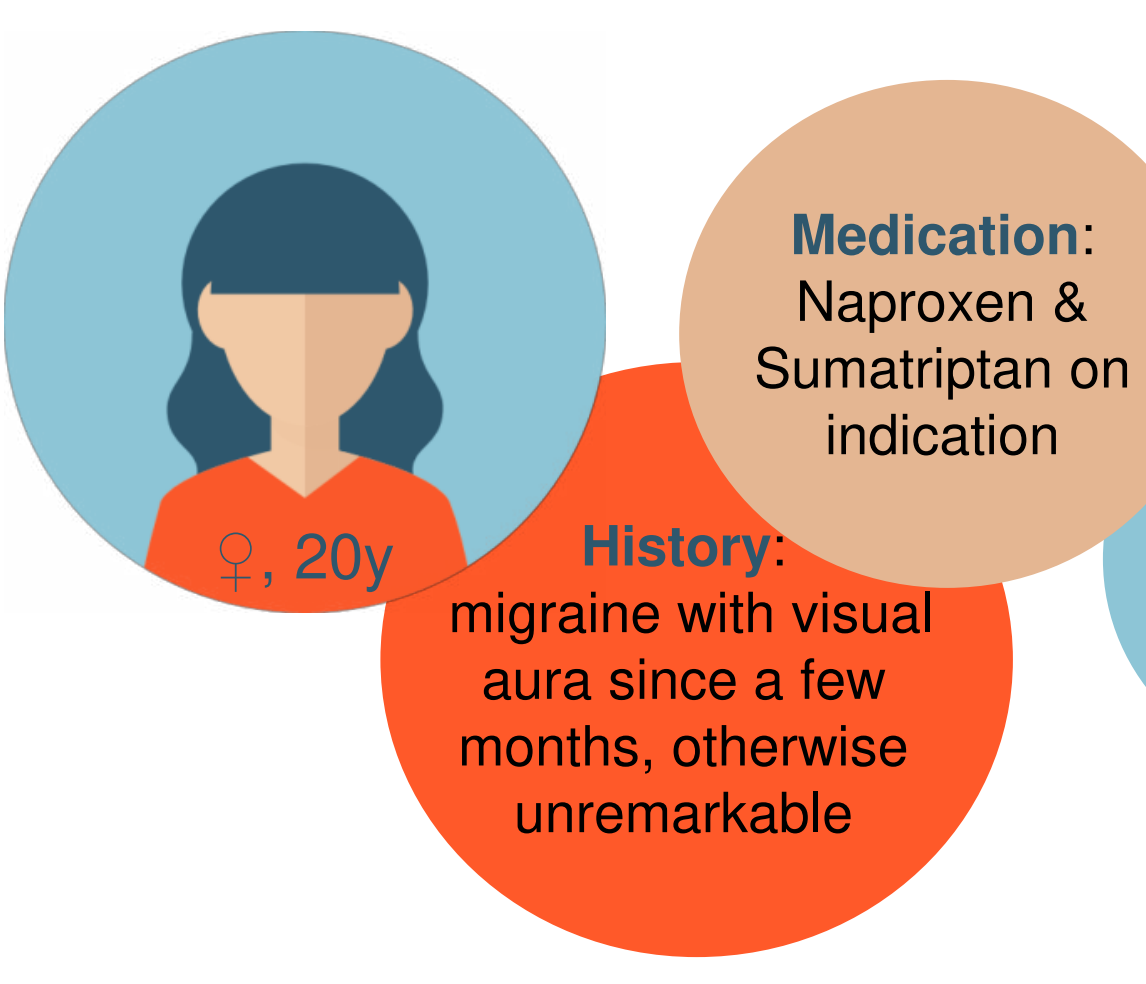

Background: Susac syndrome is a rare auto-immune disorder. Often (though not exclusively) it affects young women. Pathophysiologically, it is a non-vasculitic microangiopathy, with involvement of the small vessels of the brain, retina and inner ear, leading to headache, encephalopathy, visual disturbances and sensorineural hearing loss as key clinical symptoms ${ }^{1}$. It can cause significant morbidity if not treated adequately. Aggressive immunosuppressive treatment is therefore justified, but disease course and duration are highly variable. Treatment and disease activity monitoring is through clinical and imaging follow-up.

Methods: High resolution black-blood contrast-enhanced T1 images $^{2}$ were acquired in a patient with Susac syndrome upon diagnosis and during treatment follow-up.

Results: In our patient, there were several sulcal

Diagnosis \& treatment:

- $3 \times 1 \mathrm{~g}$ Solumedrol

- 2 x Rituximab $1 \mathrm{~g}$

- ASA $80 \mathrm{mg} / \mathrm{day}$

\section{Apr 2017: Clinically stable}

- Methylpredni $24 \mathrm{mg} /$ day

- Mycophenolate Mofetil 2 x $1 \mathrm{~g}$

- ASA 80 mg/day
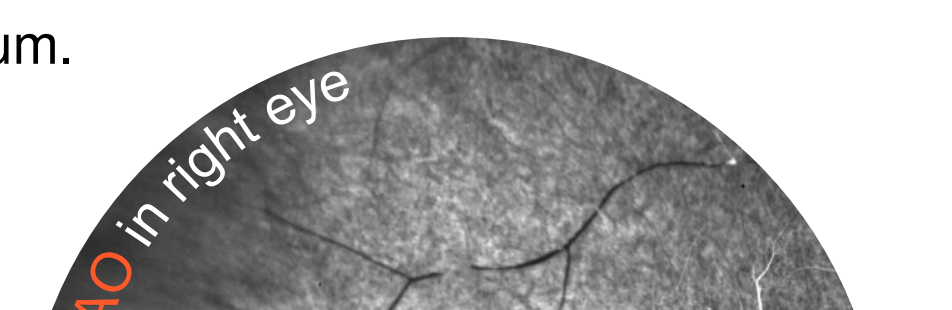

Presents Oct 2016:

of $A$
Audiogram: normal

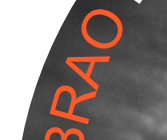

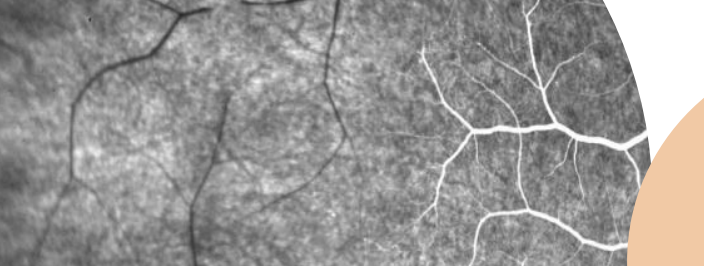

= Susac

\section{Syndrome}

